\title{
10 Jahre Radiologie Kongress Ruhr - Größter radiologischer Kongress der Region feierte Geburtstag
}

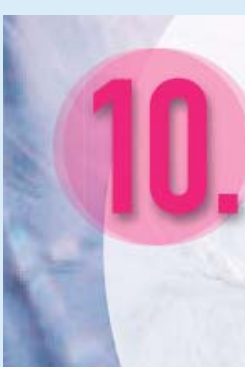

\section{RADIOLOGIE KongressRUHR}

\author{
9.- 11. Nov. 2017
RuhrCongress Bochum
}

\section{www.radiologiekongressruhr.de}

Der größte deutsche Regionalkongress der medizinischen Bildgebung feierte 2017 sein 10-jähriges Jubiläum. 1380 Radiologen, MTRA, MTRA-Schüler, Studierende und Industrievertreter fanden vom 8 . bis 11 . November ihren Weg nach Bochum und erlebten drei ereignisreiche und inhaltsstarke Kongresstage.

Prof. Dr. Lothar Heuser, einer der Gründerväter des RKR, blickte in seiner Eröffnungsrede auf die Anfänge im Jahr 2008 zurück. Damals gab es durchaus Zweifel, ob sich der RKR erfolgreich als Regionalkongress im Westen etablieren würde. Jedoch kamen schon zur Premiere über 700 Teilnehmer und mit jedem Jahr wurden es mehr. Auch das Kon-

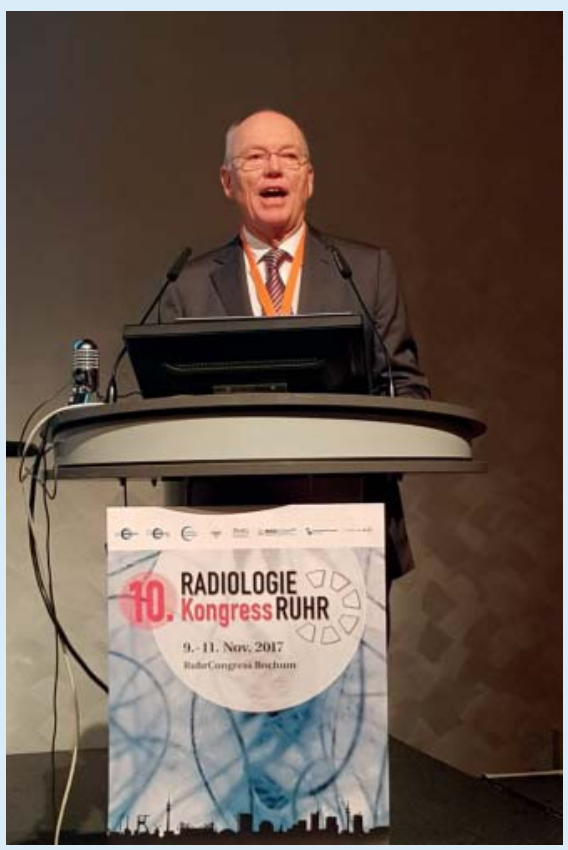

Prof. Dr. Lothar Heuser, einer der Gründerväter des RKR, eröffnete den Jubiläumskongress mit einer Rückschau auf 10 Jahre RKR. gressprogramm wurde stetig erweitert. So bietet der RKR heutzutage neben dem Hauptprogramm zu aktuellen Fortbildungsthemen zahlreiche Workshops, Zertifizierungskurse und Session für den radiologischen Nachwuchs an.

Der RKR steht seit jeher für exzellente Fortund Weiterbildung in der medizinischen Bildgebung. Dafür laden die Kongresspräsidenten jedes Jahr aufs Neue nur solche Referenten und Vorsitzende ein, die auf ihrem jeweiligen Fachgebiet federführend sind. Auch in 2017 konnten wieder herausragende Redner gewonnen werden.

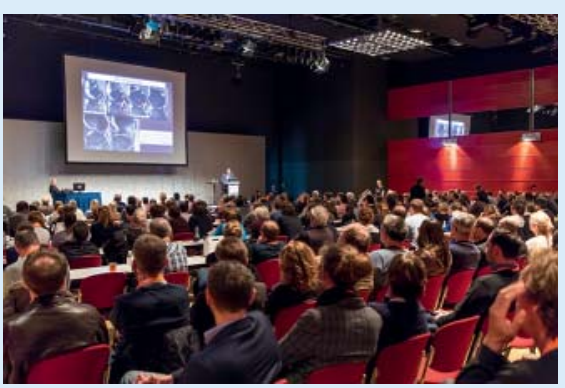

Alle Säle des RuhrCongresses waren stets gut besucht.

Anlässlich des 10. Jubilläums des RadiologieKongressRuhr hielt Prof. Dr. Claus Claussen aus Tübingen einen Highlight-Vortrag zu dem aktuellen Thema „Welche Chancen bietet die personalisierte Medizin für die Radiologie?" Die Themen IT, Künstliche Intelligenz und Big Data werden beim RKR im kommenden Jahr groß auf der Agenda stehen.

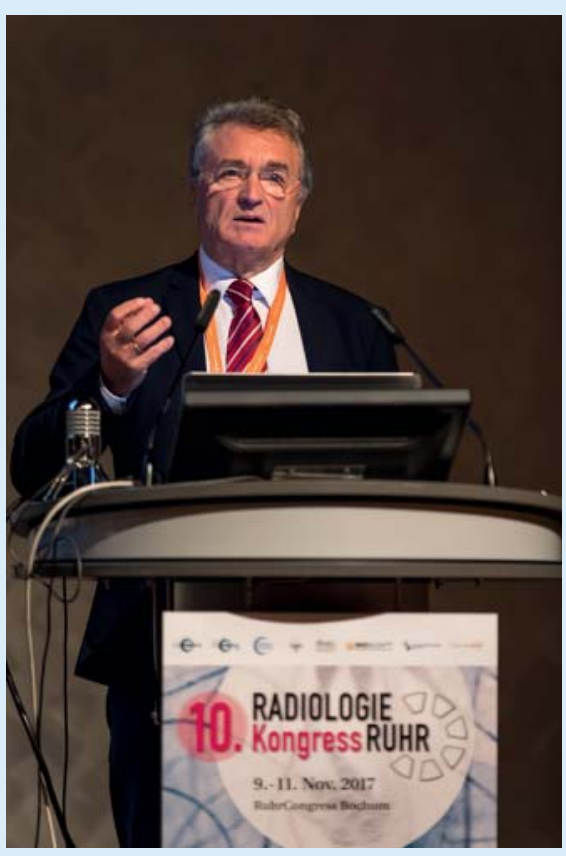

Prof. Dr. Claus Claussen aus Tübingen hielt den Highlight-Vortrag zu dem aktuellen Thema: „Welche Chancen bietet die personalisierte Medizin für die Radiologie?“.

Schon traditionell bietet der RKR Fortbildungsformate für den radiologischen Nachwuchs an. Bereits seit sechs Jahren veranstaltet die Vereinigung-Medizinisch-Technischer Berufe in der DRG (VMTB) den MTRA-Schülerdonnerstag, der jedes Jahr ausgebucht ist. Seit 2016 präsentieren zudem Schülergruppen aus fünf MTRA-Schulen aus ganz Deutschland hervorragend aufgearbeitete Vorträge für ihre Schülerkollegen. Den Preis für den besten Schülervortrag erhielt in diesem Jahr die MTRA-Schule aus Aachen. Die

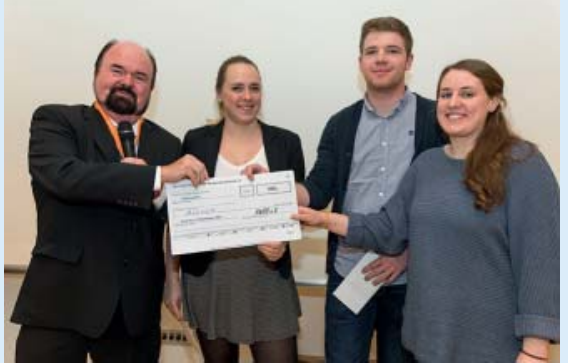

Prof. Dr. Dieter Liermann überreicht in seiner Funktion als RWRG-Präsident der MTRA-Schule aus Aachen den 1. Preis für die beste vortragende Schule. 
Rheinisch-Westfälische Röntgengesellschaft stiftete Preise für die vortragenden Schulen.

Zum 10jährigen Jubiläum fand der Abendempfang „Medizin trifft Industrie“ am ersten Kongressabend an einem ganz besonderen Ort statt: im Vonovia Ruhrstadion, ein Ort voller Tradition und Leidenschaft und das imposante Wahrzeichen der Stadt Bochum. Vor der spektakulären Kulisse des Stadions wurde das Jubiläum gefeiert und auf zehn erfolgreiche Jahre RKR angestoßen.

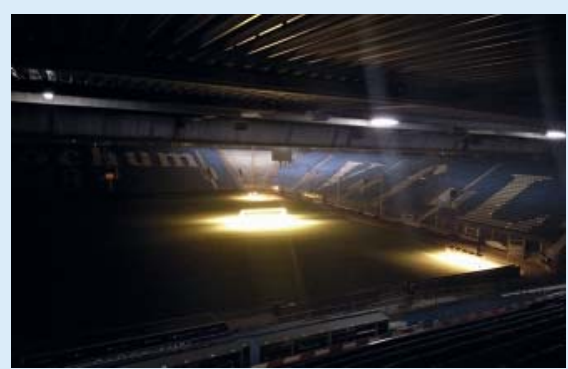

Das Vonovia Ruhrstadion bot dem Abendempfang des 10. RKR eine imposante Kulisse.

Der RadiologieKongressRuhr ist schon immer ein Kongress, der seinen Fokus auf die Fortbildung legt. Hier geht es um die Inhalte, Fertigkeiten und Techniken, die Radiologen in ihrer Praxis oder in ihrer Klinik Tag für Tag anwenden müssen. So hatten und haben die Vorträge in den Hauptsessi-

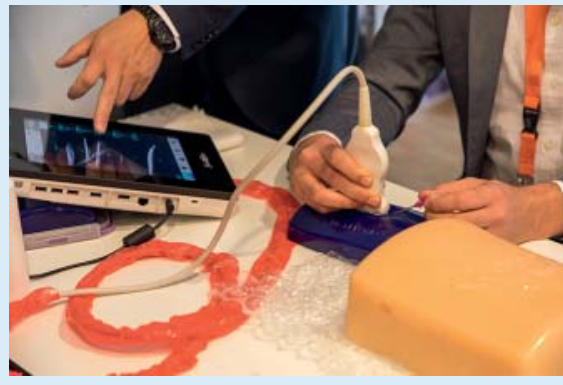

Zahlreiche Firmen präsentierten in den Hands-on-Workshops ihre technischen Neuerungen in Sachen Interventionen.

ons das Format von Refresherkursen und bauen mit dem TED-System eine sofortige Überprüfung des erlernten Wissens mit ein. Seit einigen Jahren werden diese Vorträge nun auch durch Hands-on-Workshops ergänzt, in denen an lebensnahen Simulatoren medizinische Eingriffe risikofrei trainiert werden können. Aus gleich drei verschiedenen Themenangeboten konnten die Teilnehmenden im Jubiläumsjahr 2017 wählen: Stroke, periphere Interventionen und perkutane Verfahren.

Die Industrie ist seit Anfang an ein unverzichtbarer Bestandteil des RKR. Mehr als 60 Aussteller aus dem Rhein-Ruhr-Gebiet und darüber hinaus präsentieren hier Jahr für Jahr die neuesten Entwicklungen in Technik und Forschung. In den zahlreichen Indus- triesymposien- und Workshops berichten namhafte Vertreter der Branche von den aktuellen Entwicklungen in der Radiologie. Traditionell begrüßen die Kongresspräsidenten die Vertreter der Industrie bei einem Rundgang über die Ausstellung. So ließen sich auch in 2017 Prof. Dr. Dieter Liermann, Prof. Dr. Werner Weber und Prof. Dr. Johannes Weßling die neuesten Entwicklungen und Trends präsentieren.

10 erfolgreiche Jahre RKR haben gezeigt, dass das Ruhrgebiet, die Heimat des RKR, ein ergiebiges Pflaster für die radiologische Fortbildung bietet. Zahlreiche große Kliniken und Praxen befinden sich hier und demensprechend auch viele Radiologen. Daher passt der RKR so gut dorthin und hat sich in den vergangenen zehn Jahren zu dem erfolgreichen Kongress entwickelt, der er heute ist.

2017 hat der RadiologieKongressRuhr das vorläufig letzte Mal in Bochum stattgefunden. Ab 2018 gastiert die Tagung, die unter der Schirmherrschaft der Deutschen Röntgengesellschaft und der Rheinisch-Westfälischen Röntgengesellschaft steht, erstmalig in der Dortmunder Westfalenhalle.

Weitere Informationen finden Sie unter www.radiologiekongressruhr.de. 